\title{
TRIM28-dependent SUMOylation protects the adult ovary from the male pathway
}

Authors: Moïra Rossitto ${ }^{1 \dagger}$, Stephanie Déjardin ${ }^{1}$, Chris M Rands ${ }^{2 \ddagger}$, Stephanie Le Gras ${ }^{3 \ddagger}$, Roberta Migale $^{4}$, Mahmoud-Reza Rafiee ${ }^{4}$, Yasmine Neirijnck ${ }^{2}$, Alain Pruvost ${ }^{5}$, Anvi Laetitia Nguyen ${ }^{5}$, Guillaume Bossis ${ }^{6}$, Florence Cammas ${ }^{7}$, Lionel Le Gallic ${ }^{1}$, Dagmar Wilhelm ${ }^{8}$, Robin LovellBadge $^{4}$, Brigitte Boizet-Bonhoure ${ }^{1}$, Serge $\mathrm{Nef}^{2}$, Francis Poulat $^{*}$

\begin{abstract}
Affiliations:
${ }^{1}$ Institute of Human Genetics, CNRS UMR9002 University of Montpellier, 34396 Montpellier cedex 5, France.

${ }^{2}$ Department of Genetic Medicine and Development, Faculty of Medicine, University of Geneva CMU, lab E09.2750.B 1, rue Michel-Servet CH 1211 Geneva 4 Switzerland.

${ }^{3}$ GenomEast platform, IGBMC, 1, rue Laurent Fries, 67404 ILLKIRCH Cedex, France.

${ }^{4}$ The Francis Crick Institute, 1 Midland Road, London NW1 2 1AT, UK.

${ }^{5}$ Université Paris Saclay, CEA, INRAE, Département Médicaments et Technologies pour la Santé (DMTS), SPI, 91191 Gif-sur-Yvette, France.

${ }^{6}$ Institut de Génétique Moléculaire de Montpellier (IGMM), University of Montpellier, CNRS, Montpellier, France.

${ }^{7}$ Institut de Recherche en Cancérologie de Montpellier, IRCM, INSERM U1194, Université de Montpellier, Institut régional du Cancer de Montpellier, Montpellier F-34298, France.

${ }^{8}$ Department of Anatomy and Physiology, University of Melbourne, Parkville, VIC 3010, Australia.

†Present address: Univ. Bordeaux, INRAE, Bordeaux INP, NutriNeuro, UMR 1286, F-33000, Bordeaux, France.

These authors contributed equally to this work

${ }^{*}$ Corresponding author. Email: francis.poulat@igh.cnrs.fr
\end{abstract}




\section{Summary}

Gonadal sexual fate in mammals is determined during embryonic development and must be actively maintained in adulthood. Therefore, gonadal sex-specific transcription factors are required to prevent transdifferentiation of gonadal somatic cells to the other sexual fate. Mouse genetic experiments have shown that oestrogen receptor signalling and the transcription factor FOXL2 protect ovarian granulosa cells from transdifferentiation into Sertoli cells, their testicular counterpart. However, the mechanism underlying this protective mechanism is unknown. Here, we show that one post-translational modification (i.e. SUMOylation catalysed by TRIM28) is sufficient to prevent female-to-male sex reversal of the mouse ovary after birth. We found that upon loss of TRIM28 SUMO-E3 ligase activity, ovarian granulosa cells transdifferentiate to Sertoli cells through an intermediate cell type different from gonadal embryonic progenitors. TRIM28 binds to chromatin close to the critical transcription factor FOXL2 to maintain the female pathway through SUMOylation of specific chromatin regions. Therefore, FOXL2 signalling might maintain the adult ovary cell fate via TRIM28-dependent SUMOylation. Improper SUMOylation of chromatin regions in granulosa cells might lead to female reproductive disorders and infertility, the incidence of which is currently increasing. 
For long time, it was thought that in mammals, adult gonadal sex assignment was determined and fixed during embryonic development. Any perturbation during this period leads to various disorders of sexual development. However, some teleost fish species display sequential hermaphroditism: gonadal sex is not definitively established in adulthood, and social stimuli can re-assign gonads to the opposite sex (for review see ${ }^{1}$ ). Moreover, postnatal sex-reversal has been observed in several mouse models: ovarian masculinization upon deletion of estrogen receptor 1 and $2\left(\right.$ Esr 1-2) ${ }^{2}$ or of Cyp19al ${ }^{3}$, as well as after postnatal conditional knock-out (cKO) of FoxL2 ${ }^{4}$ and ectopic ovarian expression of $\operatorname{Dmrt1}^{5}$. In these cases, the initial cellular event is the femaleto-male transdifferentiation of the supporting cell lineage (granulosa cells to Sertoli cells). Conversely, deletion of Dmrt1 in postnatal testes ${ }^{6}$ or of both $\operatorname{Sox} 8$ and $\operatorname{Sox} 9^{7}$ induces Sertoli-togranulosa cell transdifferentiation. These results indicate that granulosa and Sertoli cells retain the ability to transdifferentiate into the opposite sexual fate, and that constant repression of the alternative fate in adult life is required to maintain their cell fate identity and function. However, there is only limited information on the epigenetic and transcriptional programs implicated in cell fate reprogramming of the supporting lineage.

We previously showed that the epigenetic regulator TRIM28 is a protein partner of SOX9 in mouse fetal Sertoli cells ${ }^{8}$. However, cKO of Trim 28 in Sertoli cells, using the Amh:Cre transgenic line, results in adult males with hypoplastic testes but no sex reversal ${ }^{9}$. As $\operatorname{Trim} 28$ is expressed also in ovarian pre-granulosa cells (fig. S1), we decided to study its role in primary female sex determination by cKO of Trim 28 in pre-granulosa cells using the Nr5al:Cre transgenic line ${ }^{10,11}$. At birth, XX Trim28floxflox; Nr5al:Cre pups (Trim28 $8^{c K O}$ or $c K O$ in the text/figures) displayed normal external female genitalia, without any obvious ovarian structure abnormality at 3 days postpartum (3 dpp) (fig. S2). In FOXL2-positive immature granulosa cells, we did not detect TRIM28 
and also SOX8 and SOX9, two Sertoli cell markers, indicating that Trim28 expression in pregranulosa cells is not required for primary sex determination in females (fig. S2). However, in several follicles of 20 dpp ovaries, we observed SOX8 expression in groups of cells that stopped expressing FOXL2 (Fig. 1A). Double immunostaining showed that some SOX8-positive cells also expressed SOX9, suggesting that SOX8 expression precedes SOX9, unlike what observed in mouse embryonic testes ${ }^{12}$. As SOX8 and SOX9 are Sertoli cell markers, this suggests that fetal deletion of Trim 28 in pre-granulosa cells might induce their reprogramming towards Sertoli cells after birth, as described for Foxl2 deletion ${ }^{4}$. In 8 -week-old Trim $28^{c K O}$ mice, ovarian organization was more profoundly changed. The medullar follicles stopped expressing FOXL2, started expressing SOX8 and SOX9, and were reorganized into pseudo-tubular structures, indicative of a process of testis cord-formation. However, cortical follicles still expressed FOXL2, although some cells displayed nuclear SOX8/SOX9 expression (fig. S3). These results demonstrate that granulosa-to-Sertoli cell transdifferentiation is a two-step process that starts in follicles located in the medullar region and then spread to the cortical regions, as observed during fetal differentiation of pre-granulosa cells ${ }^{13}$. In 4-month-old Trim $28^{c K O}$ females, the transdifferentiation of granulosa cells into Sertoli cells was complete: FOXL2 expression has disappeared, and follicles were completely remodeled into tubular structures with cells that expressed the Sertoli cell markers SOX8, SOX9 and DMRT1 (Fig. 1B). Histological analysis confirmed the progressive reorganization of ovarian follicles into tubular structures and the transdifferentiation of granulosa cells into cells with a Sertoli cell morphology (fig. S4). This reorganization, which was undetectable in 4-week-old Trim $28^{c K O}$ ovaries, was clearly visible in the medulla at 8 weeks and was completed at 17 weeks. 
We next examined the temporal expression of several genes with known roles in male and female sex determination in 0.5 - (15 dpp) to 4-month-old ovaries. Reverse transcription quantitative realtime polymerase chain reaction (RT-qPCR) analysis revealed that in $\operatorname{Trim} 28^{c K O}$ ovaries, the mRNA level of most female-specific genes was decreased but for Rspol (Fig. 1C, panel Ovarian genes), whereas male-specific genes were progressively upregulated (Fig. 1C, panel Testicular genes), confirming the histology and immunofluorescence observations. To complete the transcriptomic analysis, we performed bulk RNA-seq experiments using 7-month-old Trim2 $8^{c K O}$ ovaries (Data S1), when transdifferentiation was completed. We found that 1669 genes were significantly downregulated in the absence of Trim 28 , among which $71 \%$ are normally expressed in adult granulosa cells ${ }^{14}$, including well-characterized genes involved in female sex determination (Fig. 1D, right). Repression of the granulosa cell transcriptome was accompanied by upregulation of 2897 genes that included typical Sertoli and Leydig cell markers (Fig. 1D left), showing that Trim $28 \mathrm{cKO}$ induces the ovarian transcriptome masculinization. We concluded that Trim 28 deletion in fetal pre-granulosa cells induces the postnatal remodeling of the ovarian transcriptome, leading to its masculinization.

As multiple genes involved in steroidogenesis displayed a modified profile (Fig. 1C, fig. S5A for temporal analysis, and B for RNA-seq data), we used mass spectroscopy to quantify the production of major steroid hormones in control and Trim $28^{c K O}$ ovaries and control testes from 7-month-old animals (Fig. 1E). We detected similar high levels of androgens (testosterone and androstenedione) in Trim $28^{c K O}$ ovaries and control testes. Among the estrogens produced in Trim $28^{c K O}$ ovaries, estrone was strongly reduced, whereas $17 ß$-estradiol levels were comparable to those in control ovaries. This can be explained by the persistent expression of Cyp19al (the gene encoding the aromatase that catalyzes $17 ß$-estradiol production) in Trim $28^{c K O}$ ovaries (Fig. 1C) and also by the modified expression of genes encoding hydroxysteroid dehydrogenases (HSD) (Fig. S5). Overall, 
our results indicate that fetal Trim 28 deletion induces the masculinization of the steroid production profile in adult ovaries.

To better describe the transdifferentiation process we performed single-cell RNA sequencing (scRNA-seq) to compare the transcriptomic atlas of gonadal cell types in Trim $28^{c K O}$ ovaries, control ovaries, and control testes. We chose to analyze 8-week-old gonads because our data (fig. S3) indicated that at this stage, Trim $28^{c K O}$ ovaries contain mixed population of pseudo-Sertoli cells and apparently normal granulosa cells. Using the 10X Genomics system, we analyzed 7,292 cells from Trim $28^{c K O}$ ovaries, 7,051 from control ovaries, and 42,720 from control testes (total=57,063 cells). A larger number of testis cells was required to sequence an equivalent number of male somatic cells alongside the abundant spermatogenic cells.

We catalogued the different cell populations present in all samples (fig. S6A) based on the expression of known markers (fig. S6B). We confirmed the substantial decrease of Trim 28 expression in Trim $28^{c K O}$ ovarian cells (fig. S7). In control gonads, we detected the expected cell types, including supporting (granulosa/Sertoli), steroidogenic (theca/Leydig), stroma, spermatogenic, endothelial, immune and blood cells (fig. S6), consistent with previous single-cell transcriptomic studies of adult mouse/human testis/ovaries ${ }^{15-17}$. We then focused on the supporting cell lineages. We identified 3,106 supporting cells that expressed granulosa and/or Sertoli cell markers ( $\mathrm{n}=1,112$ in Trim $28^{c K O}$ ovaries, $\mathrm{n}=1,446$ in control ovaries, and $\mathrm{n}=548$ in control testes) (Fig. 2A). In Trim $28^{c K O}$ ovaries, some supporting cells were grouped with control granulosa cells and expressed Esr2, Amh, Foxl2, Wnt4, Hsd17b1, and Nr5a2, indicating that they still had a granulosa-like transcriptome (Fig. 2B). However, we also observed a gradient of gene expression from granulosa cells to Sertoli cells via some intermediate mutant cells (Fig. 2A). These 
intermediate Trim $28^{c K O}$ ovarian supporting cells expressed some Sertoli markers at various levels and at different stages of transdifferentiation. For example, Cldn11 and Ptgds were expressed earlier during transdifferentiation and in more cells, compared with Gata1, Dmrt1, Sox9 and Sox 8 (Fig. 2C).

We then asked whether these intermediate cells resembled embryonic XX or XY supporting cell progenitors ${ }^{18}$ that had de-differentiated from granulosa cells before differentiating into the Sertoli lineage, or whether they represented a distinct cellular state between granulosa and Sertoli cells. We aligned all single cells along a pseudo-time (Fig. 2D, 2E, fig. S8) ${ }^{19}$, and divided them in three clusters based on their transcriptional profiles (Fig. 2A, right). This allowed us to identify genes that were upregulated in the granulosa, intermediate, and Sertoli cell populations (Fig. 2D, Data S3). Analysis of the mean expression of 1,743 supporting progenitor cell markers ${ }^{18}$ showed that they were weakly expressed in intermediate cells (Fig. 2E). This indicated that this population is distinct from embryonic progenitors. Gene Ontology analysis of the genes expressed in the intermediate population gave only general terms, such as "response to stimulus", "cell death", and "cell differentiation" (Data S4). Overall, the scRNA-seq analysis showed that Trim28 cKO leads in the adult mouse ovaries to transdifferentiation of the supporting lineage from the granulosa to the Sertoli cell fate. Moreover, granulosa cells do not transdifferentiate into Sertoli cell by returning to an embryonic progenitor state, but via a different and novel cellular intermediate (Fig. $2 \mathrm{~F})$.

As the Trim $28^{c K O}$ phenotype was similar to that of mice after Foxl2 deletion in adult ovarian follicles ${ }^{4}$, we asked whether these two proteins co-regulated common target genes in the ovary. Immunofluorescence analysis confirmed that TRIM28 and FOXL2 were strongly co-expressed in 
the nucleus of adult control follicular granulosa cells and to a lesser extent in theca stromal cells and both were almost undetectable in Trim $28^{c K O}$ ovaries (Fig. 3A). Next, we performed TRIM28 and FOXL2 chromatin immunoprecipitation (ChIP) followed by next generation sequencing (ChIP-seq) in control ovaries to gain a global view of TRIM28 and FOXL2 co-localization genome-wide. Comparison of the heatmaps of their co-binding to chromatin (Fig. 3B) showed that in ovaries, FOXL2 ChIP-seq reads strongly mapped to regions occupied by TRIM28 (Fig. 3B, panel a). Similarly, when we used TRIM28 ChIP-seq reads and FOXL2 peaks as reference (Fig. 3B, panel c), we observed a strong overlap between TRIM28 and FOXL2 (62\% and 55\% respectively, Fig. 3B pie charts $\mathrm{b}$ and d), confirming that these proteins share common genomic targets. TRIM28 and FOXL2 bound to overlapping regions of genes that have a central role in female sex determination, such as FoxL2, Esr2, Fst (Fig. 3C), and of genes expressed in granulosa cells (Fig. S9). As these genes were downregulated in Trim $28^{c K O}$ ovaries, this suggests that TRIM28 and FOXL2 positively regulate major granulosa cell genes. Of note, $52 \%$ of the genes downregulated in Trim $28^{c K O}$ ovaries interacted with TRIM28 and FOXL2 in control ovaries (Fig. 3D). Similarly, many male-specific genes upregulated in Trim $28^{c K O}$ ovaries were bound by TRIM28 and FOXL2 (41\%, 1,189 of 2,897), suggesting that TRIM28 and FOXL2 may have a repressive effect on the transcriptional activities of these genes in wild type ovary (Fig. 3D and fig. S10). For example, within the 2-Mb gene desert surrounding the Sox 9 gene, we observed TRIM28 and/or FOXL2 peaks in close proximity of some of the many enhancers implicated in gonadal Sox 9 expression regulation ${ }^{20}$, and also in the proximal promoter and gene body (Fig. 3C, lower panel). Similarly, the distal upstream regions of Dmrt1 and Ptgds, which are both upregulated in Trim $28^{c K O}$ ovaries, displayed overlapping regions of TRIM28 and FOXL2 binding (Fig. 3C, lower panels), like other genes, such as Cldn11 that is expressed in Sertoli cells and upregulated in Trim $28^{c K O}$ ovaries (fig. S10). 
We also analyzed DNA motif enrichment for the binding sites of the major granulosa-specific transcription factors (FOXL2 ${ }^{4}, \mathrm{RUNX}^{11}$ and ESR1/2 ${ }^{2}$ ) in TRIM28 and FOXL2 ChIP-seq data, as previously described ${ }^{8}$. We observed a significant enrichment for these motifs in regions bound by TRIM28 and FOXL2 in the ovary compared with regions bound by TRIM28 in bone marrow ${ }^{21}$ and thymus ${ }^{22}$ (Fig. 3E). This shows that in adult ovaries, both TRIM28 and FOXL2 bind to regions that display a genomic signature with binding sites for major female-specific transcription factors. To confirm that TRIM28 and FOXL2 co-localized on chromatin, we performed FOXL2 ChIP and selective isolation of chromatin-associated proteins (ChIP-SICAP) followed by mass spectrometry that provides only information relative to on-chromatin interactions ${ }^{23}$. We obtained a list of proteins located in close proximity of FOXL2 on ovarian chromatin that we ranked by abundance. As predicted, TRIM28 was amongst the top 20 FOXL2 interactors, confirming that it binds to chromatin regions very close to FOXL2 (Fig. 3F, left). A gene ontology analysis of this proteins list (that will be analyzed and published elsewhere) showed that they were mainly nuclear and chromatin factors, with only $3 \%$ of potential contaminants, demonstrating the technique specificity (Fig. 3F, right). These results are supported by a previous proteomic analysis of murine granulosa and pituitary-derived cell lines showing that TRIM28 and FOXL2 are engaged in common protein complexes ${ }^{24}$. Overall, the previous data on FOXL2 ${ }^{4}$ and our results show that in the ovary, TRIM28 and FOXL2 are implicated in the same genetic pathway to maintain the ovarian cell fate. On chromatin, this is achieved through their colocalization on regulatory regions of genes that control the granulosa and Sertoli cell fates. Our data suggest that the TRIM28 /FOXL2 pathway supports the granulosa cell fate by maintaining the female identity and suppressing the male identity. 
TRIM28 acts as a SUMO-E3 ligase by interacting with the SUMO-E2 conjugating enzyme UBC9 (encoded by the Ube2i gene) via the Plant homeodomain (PHD) and can self-SUMOylate ${ }^{25}$. SUMOylation is a reversible post-translational modification of proteins by a small ubiquitinrelated modifier (SUMO1-SUMO2/3) that involves E1, E2 and E3 enzymes (Fig. 4A). SUMOylation is involved in transcriptional regulation and regulates the activity and/or stability of many transcription factors, such as FOXL2 ${ }^{26}, \mathrm{ESR}_{2}{ }^{27}, \mathrm{NR} 5 \mathrm{~A} 1^{28}, \mathrm{GATA}^{2}{ }^{29}$, and of many chromatin-associated proteins ${ }^{30}$. TRIM28-dependent SUMOylation has been described for other proteins, such as PCNA ${ }^{31}$, CDK9 ${ }^{32}$, NPM1/B23 ${ }^{33}$, IRF7 ${ }^{34}$, VPS34 ${ }^{35}, \alpha$-synuclein, and tau ${ }^{36}$. To study in vivo the role of TRIM28-dependent SUMOylation, we generated a point mutation in exon 13 of mouse Trim 28 within the PHD domain of the TRIM28 protein (C651F) that abrogates its SUMO-E3 ligase activity ${ }^{33}$ (fig. S11). Trim2 $8^{C 651 F /+}$ heterozygous mice reproduced normally and did not show any obvious phenotype. As heterozygous mice for the Trim 28 cKO (Nr5a1:Cre;Trim28flox/+ $)$ have no phenotype, we generated Nr5a1:Cre;Trim28C651Fflox mice (Trim28 $8^{P H D / c K O}$ ). First, we showed that the TRIM28 $8^{\mathrm{C} 651 \mathrm{~F}}$ mutant protein was effectively produced and localized in the nucleus in Trim $28^{P H D / c K O}$ mutant ovaries (fig. S12). RT-qPCR analysis of 8week-old ovaries (Fig. 4B) showed that Trim 28 mRNA level in Trim $28^{P H D / c K O}$ ovaries is intermediate between control $\left(\operatorname{Trim} 28^{+/+}\right)$and Trim $28^{c K O}$ ovaries, confirming the presence of transcripts for TRIM28 $8^{\mathrm{C} 651 \mathrm{~F}}$. Moreover, female- and male-specific genes (FoxL2, Esr2, Wnt4, Hsd3b1, Ihh, and Sox9, Sox8, Dmrt1, Gata1, L-Pgds, respectively) in Trim $28^{+/+}$and Trim28PHD/+ ovaries displayed similar expression levels, showing no dominant effect of the mutated allele. Conversely, in Trim $28^{P H D / c K O}$ ovaries, ovarian genes were strongly downregulated and malespecific genes were upregulated, like in Trim $28^{c K O}$ ovaries. This suggests that Trim $28^{P H D / c K O}$ and Trim $28^{c K O}$ ovaries display a similar phenotype. Next, we compared by immunofluorescence analysis, the expression of testis markers (SOX9, SOX8, and DMRT1) and of FOXL2 in 
Trim $28^{P H D / c K O}$, Trim $28^{c K O}$, and control ovaries. Like in Trim $28^{c K O}$ ovaries, FOXL2 expression was undetectable, whereas we observed expression of the Sertoli cell markers SOX9, SOX8 and DMRT1 within structures organized in pseudo-tubules in Trim $28^{P H D / c K O}$ ovaries (Fig. 4C). Histological analysis (fig. S13) also showed a similar tissue organization in $\operatorname{Trim} 28^{P H D / c K O}$ and $\operatorname{Trim} 2^{c K O}$ ovaries. Altogether, these results indicate that maintenance of the female pathway in the adult ovary depends on the E3-SUMO ligase activity of TRIM28.

To determine whether the global SUMOylation level in the nucleus of granulosa cells was affected in $\operatorname{Trim} 28^{P H D / c K O}$ and $\operatorname{Trim} 2^{c K O}$ ovaries, we used a confocal microscopy quantitative analysis with anti-SUMO1 and -SUMO2/3 antibodies (called here SUMO2 because SUMO2 and 3 cannot be differentiated with antibodies). In both Trim $28^{P H D / c K O}$ and $\operatorname{Trim} 28^{c K O}$ ovaries, SUMO1 and particularly SUMO2 nuclear staining were decreased in ovarian somatic cells (Fig. 4D, left), as confirmed by fluorescence quantification (Fig. 4D, right). This shows that the absence of TRIM28 SUMO-E3 ligase activity in ovarian somatic cells decreased the nuclear level of SUMOylation, confirming the link between TRIM28 and this post-transcriptional modification in vivo.

To determine whether in Trim $28^{P H D / c K O}$ mice, SUMOylation was modified genome-wide, we performed quantitative SUMO1 and SUMO2 ChIP-seq analyses in adult Trim28 ${ }^{\text {PHD/cKO, }}$ Trim $28^{c K O}$ and control ovaries. We found 249,760 chromatin regions that were SUMOylated by SUMO1 or SUMO2 in control ovaries. As expected, in Trim $28^{c K O}$ and Trim $28^{P H D / c K O}$ ovaries a fraction of these peaks ( $7.3 \%$ and $5.2 \%$ respectively) displayed a significantly lower signal (Log2 FC $<-1$, AdjP value $>0.05$ ) and we designed them as hypo-SUMOylated peaks (Fig. 5A, upper panel, and blue spots in fig. S14). Of note, the number of hypo-SUMOylated peaks was higher in Trim28cKO than Trim28 $8^{P H D / c K O}$ ovaries (SUMO1+SUMO2 peaks: 18,338 versus 12,972), suggesting that the C651F mutation may not completely abolish TRIM28 E3-ligase activity, 
although it induces granulosa-to-Sertoli cell transdifferentiation, as indicated by the similar phenotype of the two mutants.

Comparison of heatmaps confirmed that SUMO1 and SUMO2 ChIP-seq reads that mapped to hypo-SUMOylated peaks were markedly decreased in Trim $28^{c K O}$ and Trim $28^{P H D / c K O}$ samples (Fig.

5A, lower panel, columns SUMO1 and 2). Moreover, TRIM28 ChIP-seq reads from control ovaries mapped strongly to these regions (Fig 5A, lower panel, column TRIM28). This shows that in control ovaries, TRIM28 occupies chromatin regions that are hypo-SUMOylated in Trim28cKO ovaries, strongly implying that TRIM28 is the E3-ligase responsible of their SUMOylation in adult ovary (either auto-SUMOylation or SUMOylation of transcription factors located near TRIM28 on chromatin). For example, many hypo-SUMOylated regions in Trim $28^{c K O}$ ovaries were occupied by TRIM28 and FOXL2 in control ovaries (fig. S15). TRIM28-dependent SUMOylation of these transcription factors might also occur before their interaction with chromatin because only a fraction (33 to 45\%) of hypo-SUMOylated regions in $\operatorname{Trim} 28^{c K O}$ and $\operatorname{Trim} 28^{P H D / c K O}$ ovaries were occupied by TRIM28 in control ovaries (fig. S15).

Surprisingly, we also found a substantial number of SUMO1 or SUMO2 peaks with a significatively stronger signal in Trim $28^{c K O}$ or Trim $28^{P H D / c K O}$ than in control ovaries (Log2 FC $>1$, AdjP val $>0.05$ ) that we designated as hyper-SUMOylated (Fig. 5B, upper panel, and red spots in fig. S14). The heatmaps showed that in $\operatorname{Trim} 28^{c K O}$ and $\operatorname{Trim} 28^{P H D / c K O}$ ovaries, hyper-SUMOylation (SUMO1 and SUMO2) occurred de novo on regions that were neither SUMOylated nor occupied by TRIM28 in control ovaries (Fig. 5B lower panel, columns SUMO1, SUMO2 and TRIM28, respectively). This was confirmed by peak analysis showing nearly no overlap between hypo- and hyper-SUMOylated regions in both mutants (fig. S16). This shows that downregulation of the female pathway in Trim $28^{c K O}$ and $\operatorname{Trim} 28^{P H D / c K O}$ ovaries allows the activation of another pathway, inducing the de novo SUMOylation of distinct chromatin regions, possibly related to the activated 
male genes. Yet, the RNA-seq analysis of Trim $28^{c K O}$ ovaries (Data S1) did not highlight the upregulation of any male-specific E3-SUMO ligase (e.g. proteins of the PIAS family), suggesting that such enzymatic activity is also expressed in granulosa cells.

Analysis of the list of hypo- and hyper-SUMOylated genes highlighted a strong correlation between the very similar phenotypes of the two mutants and gene SUMOylation. Specifically, 5,082 and 4,056 genes were hypo- and hyper-SUMOylated, respectively, in both Trim28cKO and Trim2 $8^{P H D / c K O}$ ovaries (fig. S17A). Some genes showed a mixed SUMOylation pattern (both hypoand hyper-SUMOylation peaks) (fig. S17B), suggesting a more complex regulation. However, most genes were strictly hypo- (74\%) or hyper- (75\%) SUMOylated, suggesting that they belong to distinct pathways.

Next, we analyzed the SUMOylation status of the genes identified as upregulated or downregulated in Trim $28^{c K O}$ ovaries by RNA-seq. Among the 1,669 downregulated genes (Fig. 5C, upper pie chart), the genes displaying SUMOylation variations were preferentially hypoSUMOylated (26\%), while a minority were hyper-SUMOylated (9\%) or both hypo- and hyperSUMOylated (8\%). Conversely, among the genes upregulated in Trim $28^{c K O}$ ovaries (Fig. 5C, lower pie chart), genes showing SUMOylation variations were preferentially hyper-SUMOylated (34\%), and only $8 \%$ and 7\% were hypo-SUMOylated and both hyper- and hypo-SUMOylated, respectively. Overall, TRIM28 E3-ligase controls the maintenance of granulosa cell fate via the specific SUMOylation of female genes. In its absence, a distinct pathway takes place, leading to the hyper-SUMOylation of some Sertoli cell-specific genes that is correlated with their activation. For instance, female-specific genes that were downregulated in Trim $28^{c K O}$ ovaries (Cyp1 1a1, Esr2, Foxl2, Fst, and $H s d 3 b 1$ ) displayed hypo-SUMOylated peaks in Trim $28^{c K O}$ and Trim2 $28^{P H D / c K O}$ samples for both SUMO1 and SUMO2 (Fig. 5D, upper panels, and fig. S18), where TRIM28 and FOXL2 are bound in control (Fig. 3C). Conversely, male-specific genes that were upregulated in 
Trim $28^{c K O}$ ovaries (Fig. 1 and 4) showed a mixed SUMOylation pattern (Fig. 5D, lower panel, and fig. S19). In the Sox9 locus, we observed a mixed pattern of hypo and hyper-SUMOylation in the large regulating region upstream of the gene body: four hyper-SUMOylated peaks and three hypoSUMOylated peaks in the proximity and spanning the enhancers 13,22 and $26^{20}$. Similarly, the Dmrt1 gene had two hyper-SUMOylated regions, one in the gene body and the other upstream, and one hypo-SUMOylated region. Sox 9 and Dmrt1 are key genes of the male pathway that must be strongly repressed in granulosa cells. These complex patterns of SUMOylation may reflect the genomic binding signature of transcription factors involved in $\operatorname{Sox} 9$ and $\operatorname{Dmrt} 1$ repression or activation. Conversely, Sox8 and Ptgds (like the male-specific genes presented in fig. S19) displayed only hyper-SUMOylation peaks, suggesting that SUMOylation might reflect only their transcriptional activation.

This study shows that Trim 28 plays a central role in the postnatal maintenance of the ovarian somatic cell fate. Upon Trim28 loss in fetal pre-granulosa cells, differentiated granulosa cells are reprogrammed, after birth, into Sertoli cells through a previously undescribed intermediate cell type. Unexpectedly, structural genes of Sertoli cells, such as claudin 11 (Cldn11), were upregulated before key genes encoding male transcription factors, such as $\operatorname{Sox} 9$ and $D m r t 1$. This suggests that the onset of granulosa cell transdifferentiation might not occur through the activation of a single master gene, such as $\operatorname{Sox} 9$ or Dmrt1, but through the global de-repression of the male-specific transcriptome. Our observation that TRIM28 is a co-factor of FOXL2 on chromatin supports this hypothesis. In the absence of functional TRIM28, FOXL2 would progressively loose its capacity to repress the male pathway, leading to a global de-repression of Sertoli cell genes. This process might be further accelerated by de-repression of male-pathway master genes ( $\operatorname{Sox} 9$ and $\operatorname{Dmrt} 1)$. 
Additional experiments combining single-cell ATAC-seq and RNA-seq should answer this question.

The potential role of SOX8 needs to be better investigated because our immunofluorescence and scRNA-seq experiments showed that Sox 8 is upregulated before $\operatorname{Sox} 9$ and Dmrt1 in Trim $28^{c K O}$ ovaries. Additional genetic experiments, using double Trim 28 and Sox 8 , Sox 9 or Dmrt1 knock-out lines are required to answer this question.

An important finding of our study is the role of TRIM28-dependent SUMOylation in the maintenance of granulosa cell fate. A previous study showed that global SUMOylation of chromatin-associated proteins has a key role in the stabilization of somatic and pluripotent states 37. Here we found that the TRIM28-dependent SUMOylation, which represents only a small fraction of the whole SUMOylation landscape, is sufficient to prevent adult sex-reversal. TRIM28 induces relatively sharp peaks of SUMOylation on chromatin, unlike the large peaks of histone modifications. This might reflect SUMOylated transcription factors. Therefore, a central question is the nature of TRIM28 targets. As TRIM28 can auto-SUMOylate ${ }^{25}$, a large number of hypoSUMOylated peaks in $\operatorname{Trim} 28^{c K O}$ and $\operatorname{Trim} 28^{P H D / c K O}$ ovary samples may represent TRIM28 SUMOylation; this was confirmed by the overlap between these peaks and TRIM28 peaks in control ovary samples. Similarly, many FOXL2 peaks overlapped with hypo-SUMOylated peaks in Trim $28^{c K O}$ and Trim $28^{P H D / c K O}$ ovary samples. It was previously shown that FOXL2 SUMOylation leads to its stabilization ${ }^{26}{ }^{38}$. This could explain why in Trim $28^{c K O}$ ovaries, FOXL2 is undetectable, while the transcript is still present. However, many hypo-SUMOylated peaks did not overlap with TRIM28 or FOXL2, indicating that other transcription factors or chromatinassociated proteins display TRIM28-dependent SUMOylation. SUMO-proteomics could be used to characterize these proteins. 
Our experiments show a female-related program of SUMOylation controlled by TRIM28. However, as the $\operatorname{Trim} 28^{c K O}$ and $\operatorname{Trim} 28^{P H D / c K O}$ ovarian transcriptome displays a strong masculinization, we also observed activation of a de-novo pattern of chromatin SUMOylation (i.e. hyper-SUMOylated peaks) that we attributed to the male pathway and made by an E3-ligase which remains to be determined. These hyper-SUMOylated peaks might represent SUMOylated malespecific transcription factors, such as SOX9 that can be SUMOylated ${ }^{39}$. Again, SUMO-proteomic approaches could answer this question.

Previous studies showed that transcription factors, such as FOXL2 or ESR1/2, maintain the ovarian identity and therefore its reproductive capacity. Here, we found that an enzymatic activity, TRIM28 E3-SUMO ligase activity, also is crucial for this function. Therefore, it is reasonable to hypothesize that any genetic or environmental cause that interferes with this activity might perturb ovarian function and fertility. Our findings suggest that ovarian TRIM28 could be a target of the negative effects of drugs or chemicals on human fertility and provide a potential new role for TRIM28 in ovarian pathology.

\section{References}

1 Capel, B. Vertebrate sex determination: evolutionary plasticity of a fundamental switch. Nat Rev Genet, doi:10.1038/nrg.2017.60 (2017).

2 Couse, J. F. et al. Postnatal sex reversal of the ovaries in mice lacking estrogen receptors alpha and beta. Science 286, 2328-2331, doi:10.1126/science.286.5448.2328 (1999).

3 Britt, K. L. et al. Estrogen regulates development of the somatic cell phenotype in the eutherian ovary. FASEB J 16, 1389-1397, doi:10.1096/fj.01-0992com (2002).

4 Uhlenhaut, N. H. et al. Somatic sex reprogramming of adult ovaries to testes by FOXL2 ablation. Cell 139, 1130-1142, doi:10.1016/j.cell.2009.11.021 (2009). 
5 Lindeman, R. E. et al. Sexual cell-fate reprogramming in the ovary by DMRT1. Curr Biol 25, 764-771, doi:10.1016/j.cub.2015.01.034 (2015).

6 Matson, C. K. et al. DMRT1 prevents female reprogramming in the postnatal mammalian testis. Nature 476, 101-104, doi:10.1038/nature10239 (2011).

Barrionuevo, F. J. et al. Sox 9 and Sox 8 protect the adult testis from male-to-female genetic reprogramming and complete degeneration. eLife 5, doi:10.7554/eLife.15635 (2016).

8 Rahmoun, M. et al. In mammalian foetal testes, SOX9 regulates expression of its target genes by binding to genomic regions with conserved signatures. Nucleic Acids Res 45, 7191-7211, doi:10.1093/nar/gkx328 (2017).

Herzog, M. et al. TIF1beta association with HP1 is essential for post-gastrulation development, but not for Sertoli cell functions during spermatogenesis. Dev Biol 350, 548558, doi:10.1016/j.ydbio.2010.12.014 (2011).

10 Bingham, N. C., Verma-Kurvari, S., Parada, L. F. \& Parker, K. L. Development of a steroidogenic factor 1/Cre transgenic mouse line. Genesis 44, 419-424 (2006).

11 Nicol, B. et al. RUNX1 maintains the identity of the fetal ovary through an interplay with FOXL2. Nat Commun 10, 5116, doi:10.1038/s41467-019-13060-1 (2019).

12 Schepers, G., Wilson, M., Wilhelm, D. \& Koopman, P. SOX8 is expressed during testis differentiation in mice and synergizes with SF1 to activate the Amh promoter in vitro. $J$ Biol Chem 278, 28101-28108, doi:10.1074/jbc.M304067200 (2003).

13 Niu, W. \& Spradling, A. C. Two distinct pathways of pregranulosa cell differentiation support follicle formation in the mouse ovary. Proc Natl Acad Sci US A 117, 20015-20026, doi:10.1073/pnas.2005570117 (2020).

14 Sumitomo, J. et al. Mouse oocytes suppress miR-322-5p expression in ovarian granulosa cells. J Reprod Dev 62, 393-399, doi:10.1262/jrd.2015-161 (2016). developmental mapping and $\mathrm{X}$ chromosome transcriptional dynamics during mouse spermatogenesis. Nat Commun 10, 1251, doi:10.1038/s41467-019-09182-1 (2019).

16 Fan, X. et al. Single-cell reconstruction of follicular remodeling in the human adult ovary. Nat Commun 10, 3164, doi:10.1038/s41467-019-11036-9 (2019).

17 Wagner, M. et al. Single-cell analysis of human ovarian cortex identifies distinct cell populations but no oogonial stem cells. Nat Commun 11, 1147, doi:10.1038/s41467-02014936-3 (2020).

18 Stevant, I. et al. Dissecting Cell Lineage Specification and Sex Fate Determination in Gonadal Somatic Cells Using Single-Cell Transcriptomics. Cell reports 26, 3272-3283 e3273, doi:10.1016/j.celrep.2019.02.069 (2019).

19 Haghverdi, L., Buttner, M., Wolf, F. A., Buettner, F. \& Theis, F. J. Diffusion pseudotime robustly reconstructs lineage branching. Nat Methods 13, 845-848, doi:10.1038/nmeth.3971 (2016).

20 Gonen, N. et al. Sex reversal following deletion of a single distal enhancer of Sox9. Science 360, 1469-1473, doi:10.1126/science.aas9408 (2018).

21 Barde, I. et al. A KRAB/KAP1-miRNA cascade regulates erythropoiesis through stagespecific control of mitophagy. Science 340, 350-353, doi:10.1126/science.1232398 (2013).

22 Santoni de Sio, F. R. et al. KAP1 regulates gene networks controlling T-cell development and responsiveness. FASEB J 26, 4561-4575, doi:10.1096/fj.12-206177 (2012).

23 Rafiee, M. R., Girardot, C., Sigismondo, G. \& Krijgsveld, J. Expanding the Circuitry of Pluripotency by Selective Isolation of Chromatin-Associated Proteins. Mol Cell 64, 624635, doi:10.1016/j.molcel.2016.09.019 (2016). 
24 Penrad-Mobayed, M. et al. Conventional and unconventional interactions of the transcription factor FOXL2 uncovered by a proteome-wide analysis. FASEB J 34, 571587, doi:10.1096/fj.201901573R (2020).

25 Ivanov, A. V. et al. PHD domain-mediated E3 ligase activity directs intramolecular sumoylation of an adjacent bromodomain required for gene silencing. Mol Cell 28, 823837, doi:10.1016/j.molcel.2007.11.012 (2007).

26 Georges, A. et al. SUMOylation of the Forkhead transcription factor FOXL2 promotes its stabilization/activation through transient recruitment to PML bodies. PLoS One 6, e25463, doi:10.1371/journal.pone.0025463 (2011).

Picard, N. et al. Identification of estrogen receptor beta as a SUMO-1 target reveals a novel phosphorylated sumoylation motif and regulation by glycogen synthase kinase 3 beta. Mol Cell Biol 32, 2709-2721, doi:10.1128/MCB.06624-11 (2012).

28 Lee, F. Y. et al. Eliminating SF-1 (NR5A1) sumoylation in vivo results in ectopic hedgehog signaling and disruption of endocrine development. Dev Cell 21, 315-327, doi:10.1016/j.devcel.2011.06.028 (2011).

29 Wang, J., Feng, X. H. \& Schwartz, R. J. SUMO-1 modification activated GATA4dependent cardiogenic gene activity. $J$ Biol Chem 279, 49091-49098, doi:10.1074/jbc.M407494200 (2004).

30 Wilson, N. R. \& Hochstrasser, M. The Regulation of Chromatin by Dynamic SUMO Modifications. Methods Mol Biol 1475, 23-38, doi:10.1007/978-1-4939-6358-4_2 (2016).

31 Li, M., Xu, X., Chang, C. W. \& Liu, Y. TRIM28 functions as the SUMO E3 ligase for PCNA in prevention of transcription induced DNA breaks. Proc Natl Acad Sci U S A, doi:10.1073/pnas.2004122117 (2020).

32 Ma, X. et al. TRIM28 promotes HIV-1 latency by SUMOylating CDK9 and inhibiting PTEFb. eLife 8, doi:10.7554/eLife.42426 (2019).

33 Neo, S. H. et al. TRIM28 Is an E3 Ligase for ARF-Mediated NPM1/B23 SUMOylation That Represses Centrosome Amplification. Mol Cell Biol 35, 2851-2863, doi:10.1128/MCB.01064-14 (2015).

34 Liang, Q. et al. Tripartite motif-containing protein 28 is a small ubiquitin-related modifier E3 ligase and negative regulator of IFN regulatory factor 7. J Immunol 187, 4754-4763, doi:10.4049/jimmunol.1101704 (2011).

35 Yang, Y. et al. Acetylated hsp70 and KAP1-mediated Vps34 SUMOylation is required for autophagosome creation in autophagy. Proc Natl Acad Sci U S A 110, 6841-6846, doi:10.1073/pnas.1217692110 (2013).

3536 Rousseaux, M. W. et al. Depleting Trim28 in adult mice is well tolerated and reduces levels of alpha-synuclein and tau. eLife 7, doi:10.7554/eLife.36768 (2018).

37 Cossec, J. C. et al. SUMO Safeguards Somatic and Pluripotent Cell Identities by Enforcing Distinct Chromatin States. Cell Stem Cell 23, 742-757 e748, doi:10.1016/j.stem.2018.10.001 (2018).

Kim, J. H. et al. FOXL2 posttranslational modifications mediated by GSK3beta determine the growth of granulosa cell tumours. Nat Commun 5, 2936, doi:10.1038/ncomms3936 (2014).

39 Komatsu, T. et al. Small ubiquitin-like modifier 1 (SUMO-1) modification of the synergy control motif of Ad4 binding protein/steroidogenic factor 1 (Ad4BP/SF-1) regulates synergistic transcription between Ad4BP/SF-1 and Sox9. Mol Endocrinol 18, 2451-2462 (2004).

40 Minkina, A. et al. DMRT1 Protects Male Gonadal Cells from Retinoid-Dependent Sexual Transdifferentiation. Dev Cell 29, 511-520, doi:10.1016/j.devcel.2014.04.017 (2014). 
41 Cocquet, J., Pannetier, M., Fellous, M. \& Veitia, R. A. Sense and antisense Fox12 transcripts in mouse. Genomics 85, 531-541 (2005).

\section{Acknowledgments}

We thank Nitzan Gonen from Bar-Ilan University for scientific discussions and critical reading of the manuscript. We are grateful to Monsef Benkirane and Dominique Giorgi for their continual support to the project. We thank the staff of the 'Reseau d'Histologie de Montpellier'(RHEM) for paraffin embedding of tissue samples and histology staining. We thank Marie-Pierre Blanchard, and Amelie Sarrazin of the Montpellier Imaging Facility (MRI) for their help with microscopy experiments. We thank the staff of the RAM-CECEMA animal care facility (University of Montpellier). We thank Francoise Kuhne for technical assistance in single-cell analysis. We thank the GenomEast platform, particularly Violaine Alunni for the preparation of RNA-seq libraries and Romain Kaiser for sequencing. We thank Prof Michael Wegner and Prof David Zarkower for their generous gift of the anti-SOX8 and anti-Dmrt1 antibodies respectively. We thank the Freiburg Galaxy server that was used for some calculations. We thank Elisabetta Andermarcher for manuscript editing.

\section{Funding}

This study was supported by the Centre National de la Recherche Scientifique (CNRS), the University of Montpellier, and by a grant from 'Agence Nationale de la Recherche' (ANR-16CE14-0020-SexMaintain to F.P).

R.M and R.L.B are funded by the Francis Crick Institute. The Francis Crick Institute receives its core funding from Cancer Research UK (FC001107), the UK Medical Research Council (FC001107), the Wellcome (FC001107), and by the UK Medical Research Council (U117512772). 


\section{Author contribution}

M.R and F.P designed the study. M.R, S.D, L.L and B.B.B performed histological and RT-qPCR experiments. S.N and F.P designed the scRNA-seq. C.R, MR, Y.N and S.N. performed and analyzed scRNA-seq. F.P designed and performed ChIPseq experiments. All bioinformatics

analyses, excepted for scRNA-seq, were performed by S.L. F.C designed and created the mouse line carrying Trim $28^{\text {Phd }}$ allele. A.P and A.L.N performed steroids dosage. G.B and D.W produced and purified the SUMOs and FOXL2 antibodies respectively. R.L.B, R.M and M.R.R designed, performed and analysed FOXL2-ChIP-SICAP. F.P wrote the manuscript. All authors reviewed and added input to the manuscript.

\section{Competing interests}

The authors have no competing of interests.

\section{Data and materials availability}

All data are available in the main text or the supplementary materials. RNA-seq and ChIP-seq Data have been deposited in the Gene Expression Omnibus under accession number GSE166385 (RNAseq and ChIP-seq) and GSE166444 (Single cell RNA-seq) and the mass spectrometry proteomics data have been deposited to the ProteomeXchange Consortium via the PRIDE partner repository with the dataset identifier PXD024439 
bioRxiv preprint doi: https://doi.org/10.1101/2021.03.24.436749; this version posted March 24, 2021. The copyright holder for this preprint (which was not certified by peer review) is the author/funder, who has granted bioRxiv a license to display the preprint in perpetuity. It is made available under aCC-BY-NC-ND 4.0 International license.

Fig.1

A

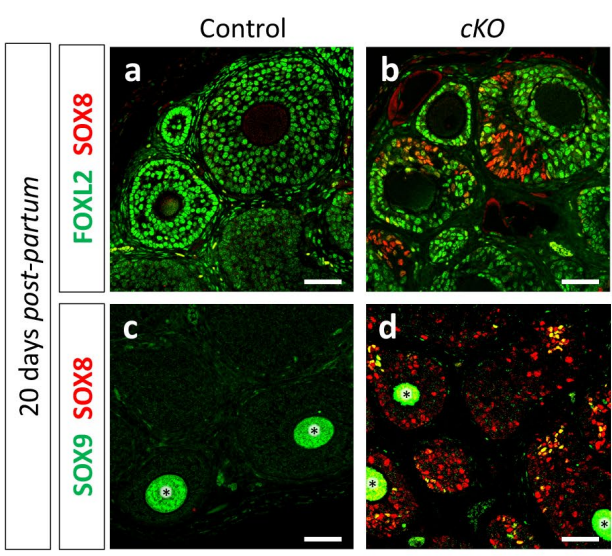

C

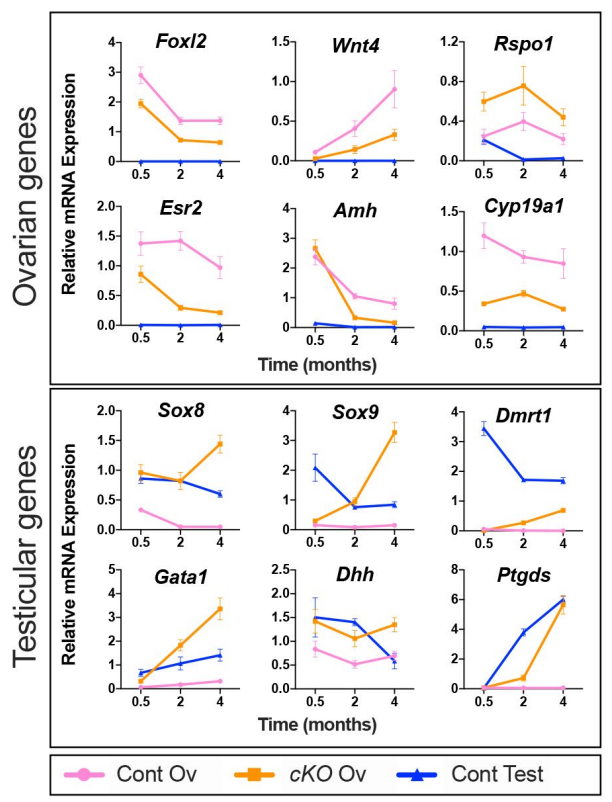

$\mathrm{E}$
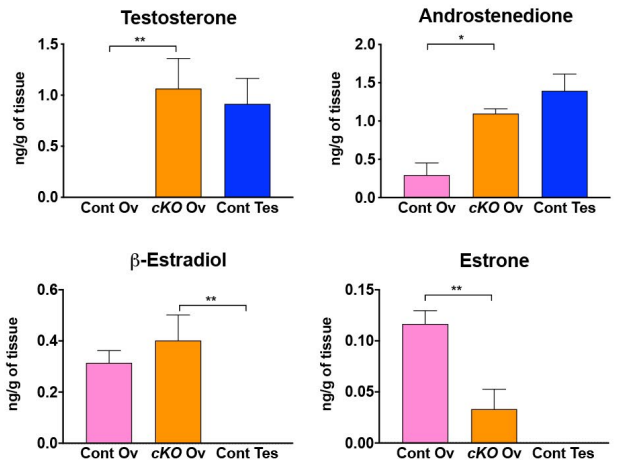

B

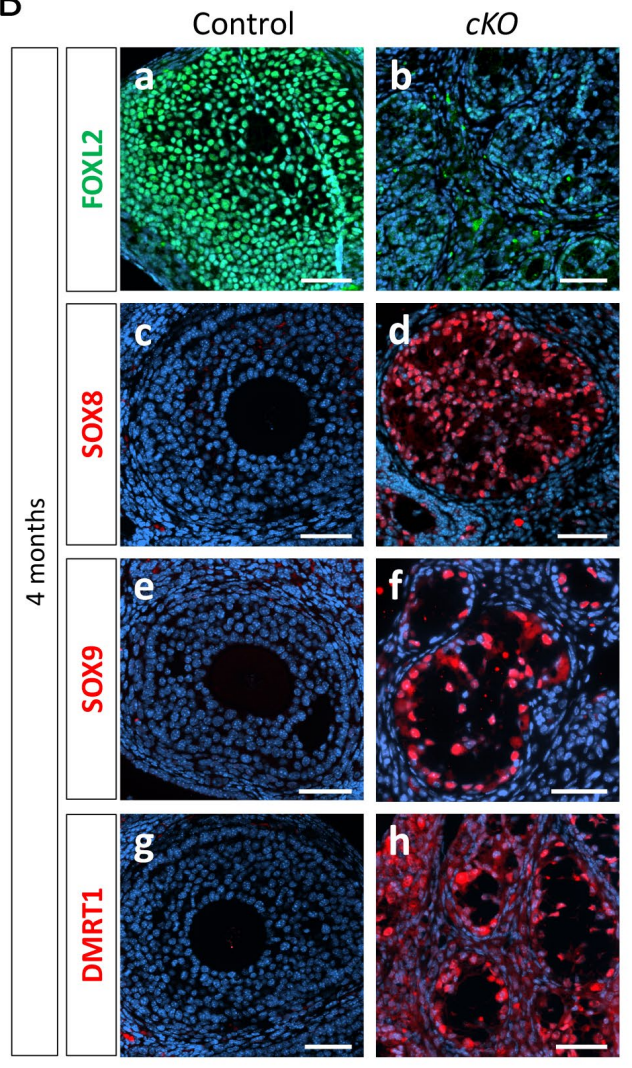

D
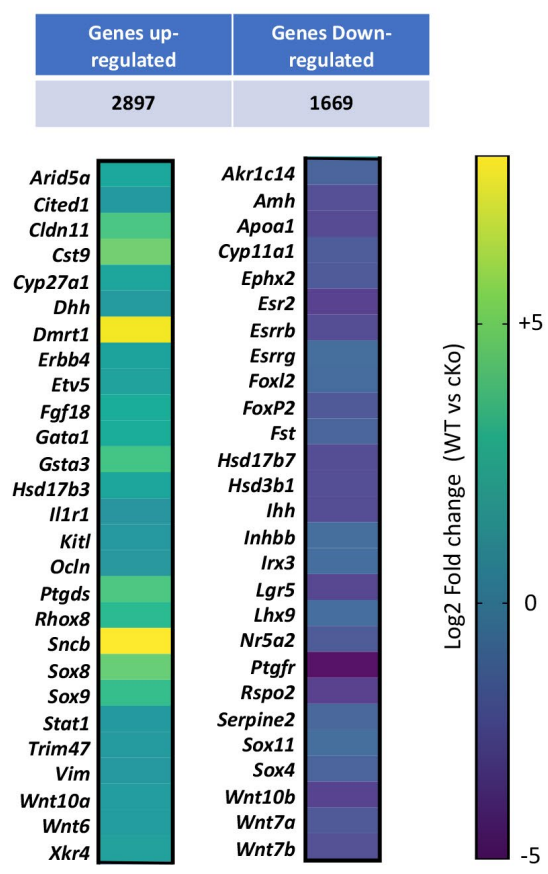
Fig.1: Trim28 loss in granulosa cells induces masculinization of the adult ovary. (A). Compared with control ovaries (a and c), in granulosa cells of 20 dpp Trim $28^{c K O}$ ovaries, FOXL2 expression is progressively lost and SOX8 (Sertoli cell marker) start to be expressed (b). Among the SOX8-positive cells, few express also SOX9 (d), suggesting that SOX8 may precede SOX9 expression onset. Green staining of oocytes $\left(^{*}\right)$ is a non-specific antibody artifact of early folliculogenesis ${ }^{40}$. Scale bar: $50 \mu \mathrm{m}$. (B). In 4-month-old Trim $28^{c K O}$ ovaries, transdifferentiation to male supporting cells is complete. Compared with control ovary (a, c, e, g), in Trim2 $8^{c K O}$ ovaries FOXL2 signal has almost disappeared (b), follicles are reorganized in pseudo-tubules that express the Sertoli markers SOX8 (d), SOX9 (f), and DMRT1 (h). Scale bar: $50 \mu \mathrm{m}$. (C). RT-qPCR analysis of the temporal (in months) gene expression variations in control ovaries (Cont Ov), Trim $28^{c K O}$ ovaries ( $c K O \mathrm{Ov}$ ), and control testes (Cont Test). In Trim $28^{c K O}$ ovaries, typical ovarian genes are progressively downregulated, but for Rspo1, and testis genes are upregulated. (D). Heatmap of the RNA-seq analysis of 7-month-old ovaries (see Data S1) showing that 2,896 and 1,669 genes are up- and down-regulated, respectively, in Trim $28^{c K O}$ compared with control ovaries. Normalized expression values are expressed in Log2 fold change (Control vs $c K O$ ), from -5 (deep violet) to +8 (yellow). (E). Trim $28 \mathrm{cKO}$ induces the masculinization of the ovarian steroid profile. Steroids were extracted from 7-month-old control (Cont Ov) and Trim2 $8^{c K O}$ (cKO Ov) ovaries, and control testes (Cont Test) and quantified (ng/g of tissue) by mass spectroscopy. Data are the mean $\pm \operatorname{SEM}(\mathrm{n}=3) ; * * P<0.005, * P<0.05$ (ANOVA with Tukey's multiple comparisons test). 
Fig. 2
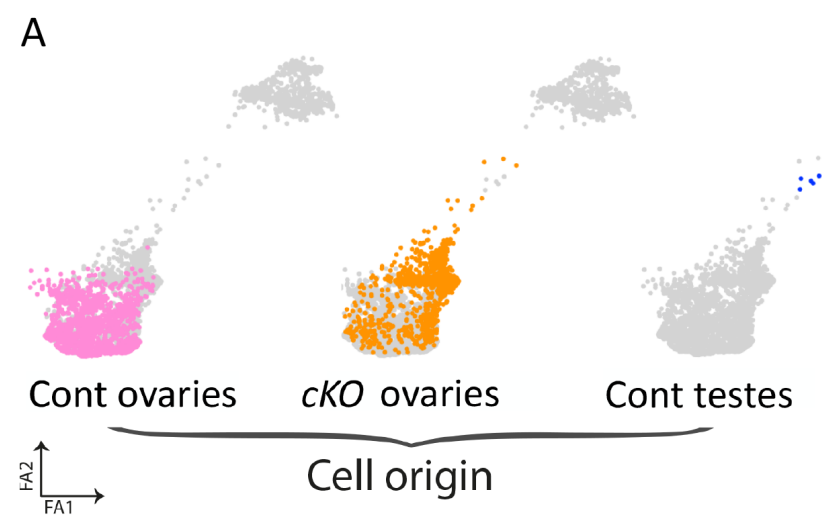

B

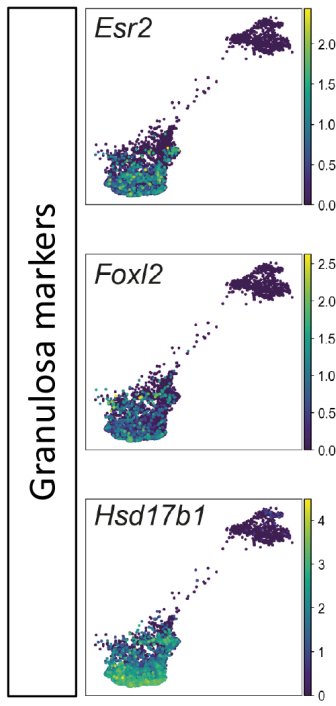

$\mathrm{C}$

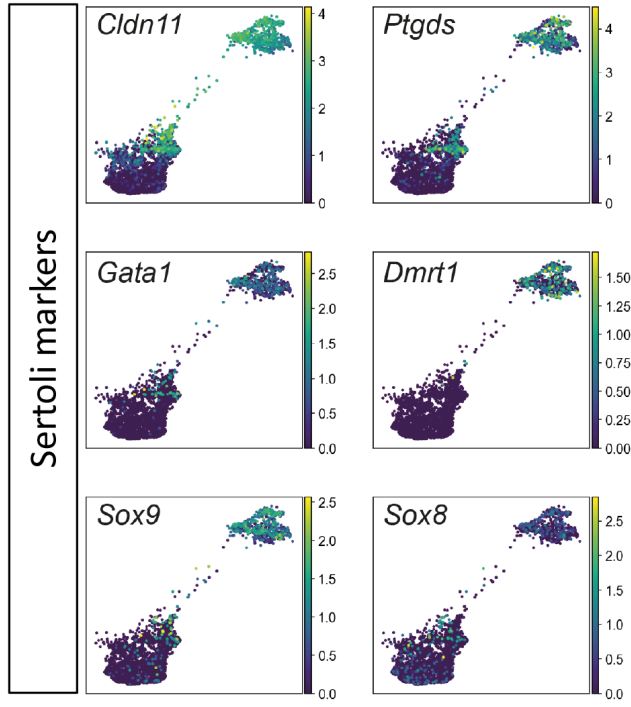

D
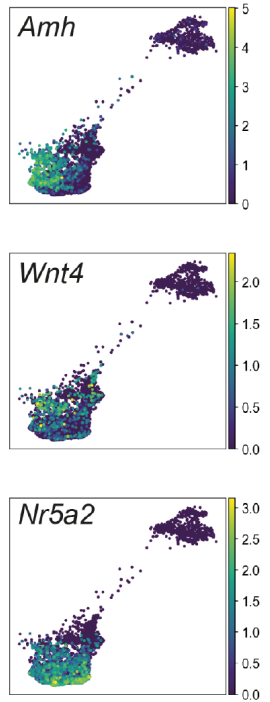

$E$
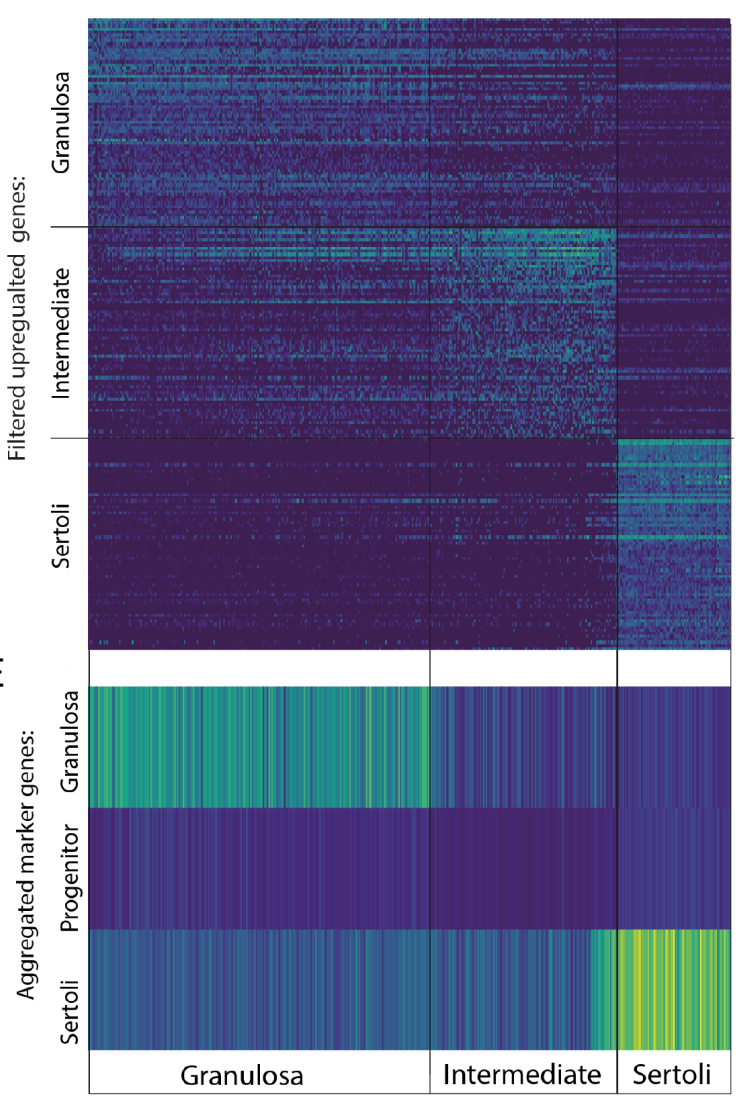

$\mathrm{F}$

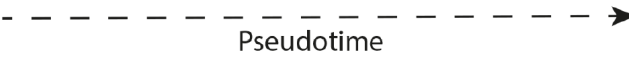

Differentiation:

Transdifferentiation:
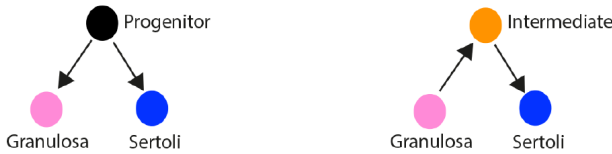
Fig. 2: scRNA-seq analysis of ovarian and testis supporting cells reveals an intermediate cell population during transdifferentiation. (A). Force directed graphs showing the scRNA-seq results of adult Trim $28^{c K O}$ ovarian supporting cells (orange), control granulosa (pink), and Sertoli cells (blue) (left). Each dot is one cell (colored according to the sample of origin), and the distance

between cells indicates their inferred transcriptional similarity. Leiden clustering divided the cells in three populations displayed using partition-based graph abstraction (right). Each node represents a cell cluster, and the proportion of $\operatorname{Trim} 28^{c K O}$, control granulosa and Sertoli cells is shown as a pie chart on each node. The edges between nodes represent the neighborhood relation among clusters with a thicker line showing a stronger connection between clusters. (B). and (C). Gene expression of selected granulosa and Sertoli cell markers in the supporting cells analyzed in (A). Each dot corresponds to one cell from (A), and gene expression level ranges from 0 (purple) to high (yellow). (D). Heatmap showing the expression level of the top filtered differentially expressed genes in the three cell clusters along the pseudo-time. See Table S3 for the full list of genes. (E). Heatmap showing the mean expression levels in the three cell clusters along the pseudo-time of several thousand genes from a previous study on the granulosa, supporting progenitor, and Sertoli cell lineages ${ }^{18}$. (F). Schematic illustrating the processes of differentiation and transdifferentiation. 
bioRxiv preprint doi: https://doi.org/10.1101/2021.03.24.436749; this version posted March 24, 2021. The copyright holder for this preprint (which was not certified by peer review) is the author/funder, who has granted bioRxiv a license to display the preprint in perpetuity. It is made available under aCC-BY-NC-ND 4.0 International license.

Fig. 3

A
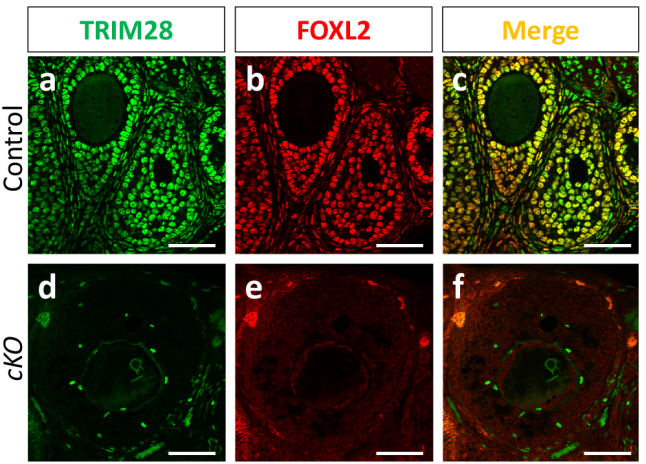

C
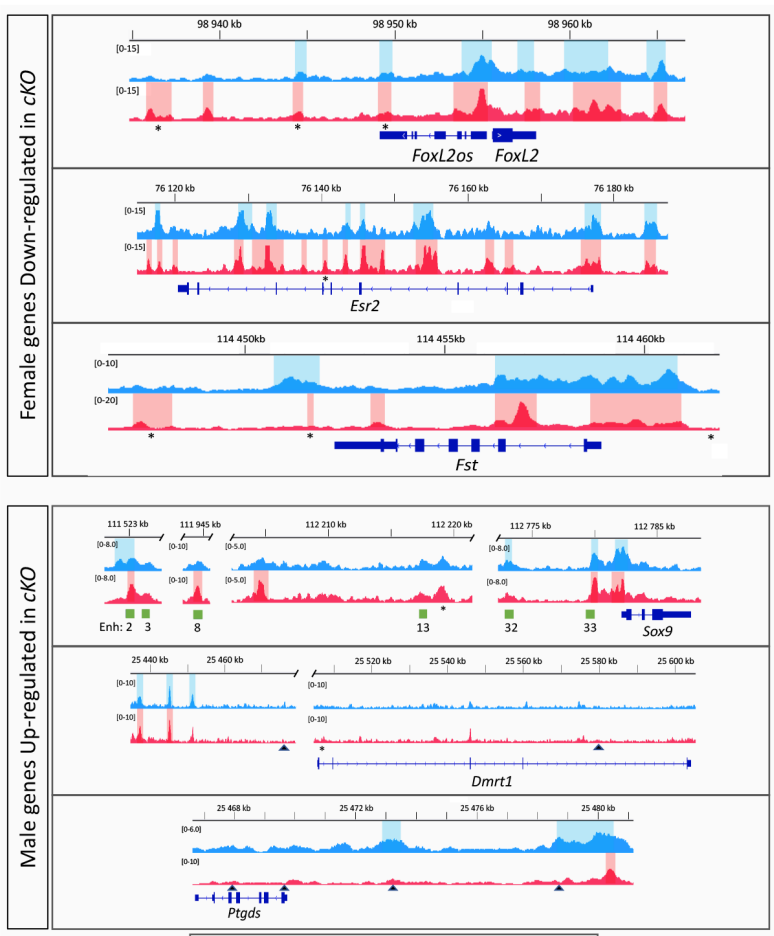

Relevant Peaks: 1 TRIM28 1 FOXL2

$\mathrm{F}$

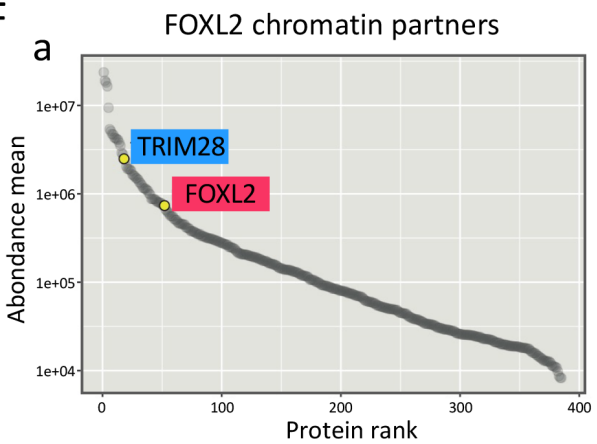

B

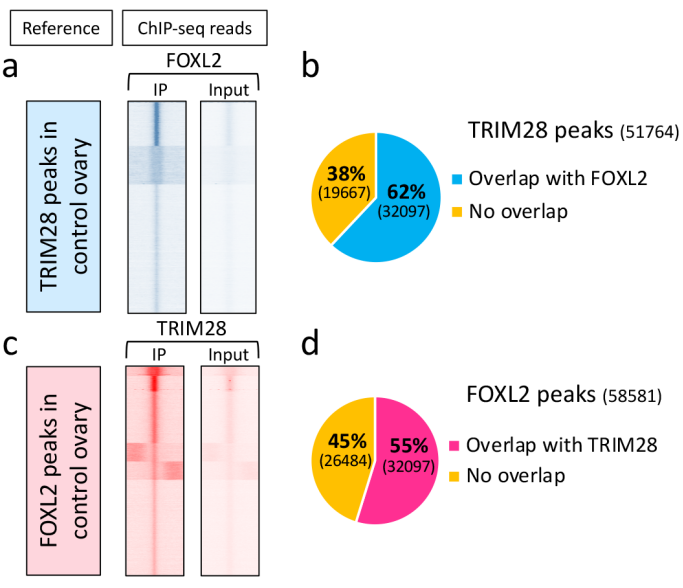

D

Genes Down-Regulated (1668)

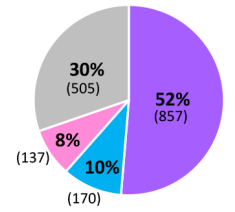

Genes Up-Regulated (2897)

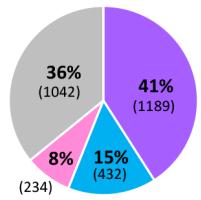

- Bound TRIM28 and FOXL2 - Bound TRIM28

= Bound FOXL2 " none

$\mathrm{E}$

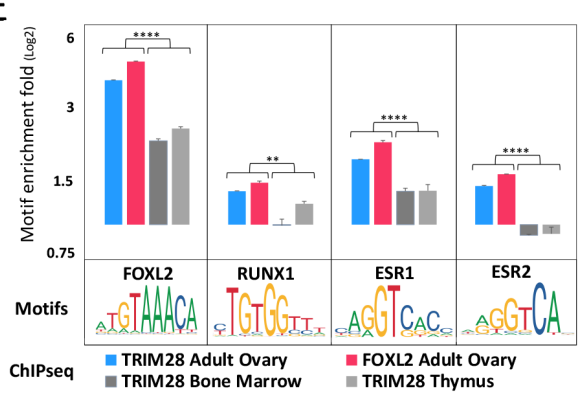

b

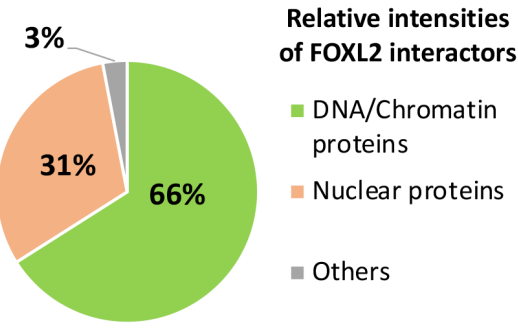




\section{Fig. 3: TRIM28 and FOXL2 act together on chromatin to maintain the female pathway.}

(A). TRIM28 and FOXL2 are co-expressed in the nucleus of most follicular granulosa cells in 4- month control ovaries $\left(\mathrm{a}, \mathrm{b}\right.$ and $\mathrm{c}$ ) and in cells with flat nucleus surrounding follicles identified as steroidogenic theca cells. In Trim $28^{c K O}$ genomic localization in adult ovary. Heatmaps a and c: blue and red traces represent FOXL2 and TRIM28 ChIP-seq reads from control (Cont) and mutant $(c K O)$ ovaries, respectively $( \pm 1 \mathrm{~kb}$ from the center of the reference TRIM28 and FOXL2 ChIP-seq peaks in control ovaries). $b$ and d: pie charts showing that 32,097 of the 51,764 TRIM28 peaks (62\% in b) and of the 58,581 FOXL2 peaks (55\% in d) overlap in control ovaries. (C). Examples of TRIM28 and/or FOXL2 peaks in/around genes the expression of which is altered in Trim $28^{\mathrm{cKO}}$ ovaries. Upper panel: female-specific genes downregulated in Trim $28^{c K O}$ ovaries (see also fig. S9). The FoxL2 gene is represented with the co-regulated non-coding FoxL2os gene ${ }^{41}$. Lower panel: male-specific genes upregulated in Trim $28^{c K O}$ ovaries (see also fig. S10). Green rectangles in the $\operatorname{Sox} 9$ panel: putative enhancers that, with enhancer 13, are crucial for sex determination ${ }^{20}$. Relevant ChIP-seq peaks are highlighted in light blue (TRIM28) and light red (FOXL2). * and $\Delta$ : regions that hypoand hyper-SUMOylated in Trim $28^{c K O}$ ovaries, respectively (see Fig 5). (D). Pie charts showing up- and downregulated genes in Trim $28^{c K O}$ ovaries that are bound by TRIM28 and/or FOXL2. Genes are listed in Data S7. (E). Enrichment for binding motifs of transcription factors involved in granulosa cell fate maintenance (FOXL2, RUNX1 and ESR1/2) in reads of TRIM28 and FOXL2 ChIP-seq of adult control ovary (this study), and TRIM28 ChIP-seq of bone marrow ${ }^{21}$ and of thymus ${ }^{22}$. (F). (a). Plot showing enriched proteins, ranked by abundance, identified by FOXL2 ChIP-SICAP. Only significant proteins ( $>2$-fold enrichment over No antibody control, $n=2$ ) are shown. TRIM28 was identified amongst the top 20 proteins found to interact with FOXL2. (b).

Percentage of the relative intensities of FOXL2 chromatin partners, normalized to the total abundance of the enriched proteins. 
Fig. 4

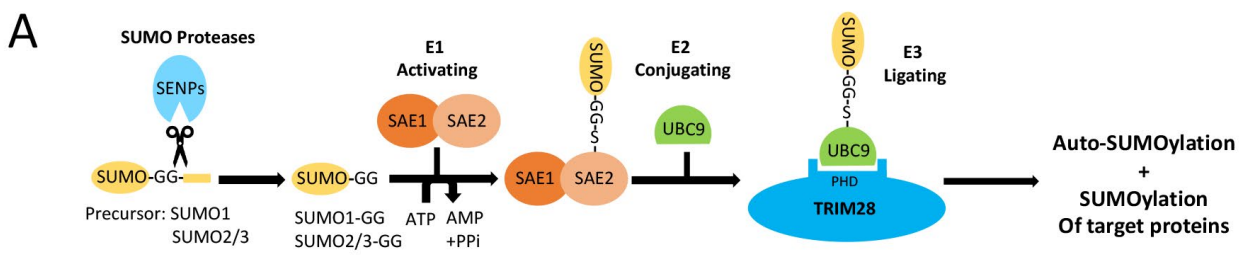

B

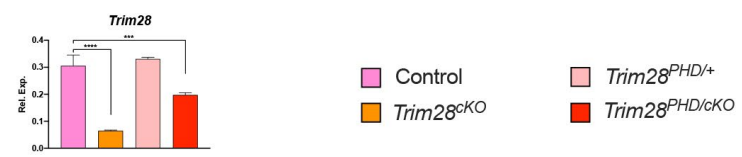

Female genes
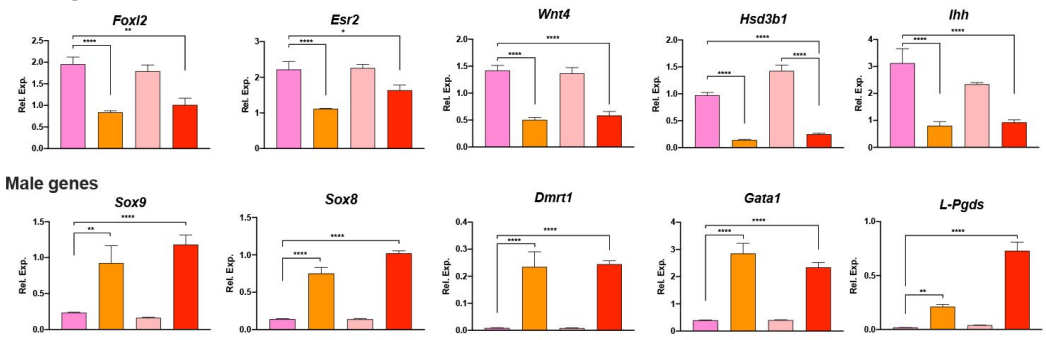

C
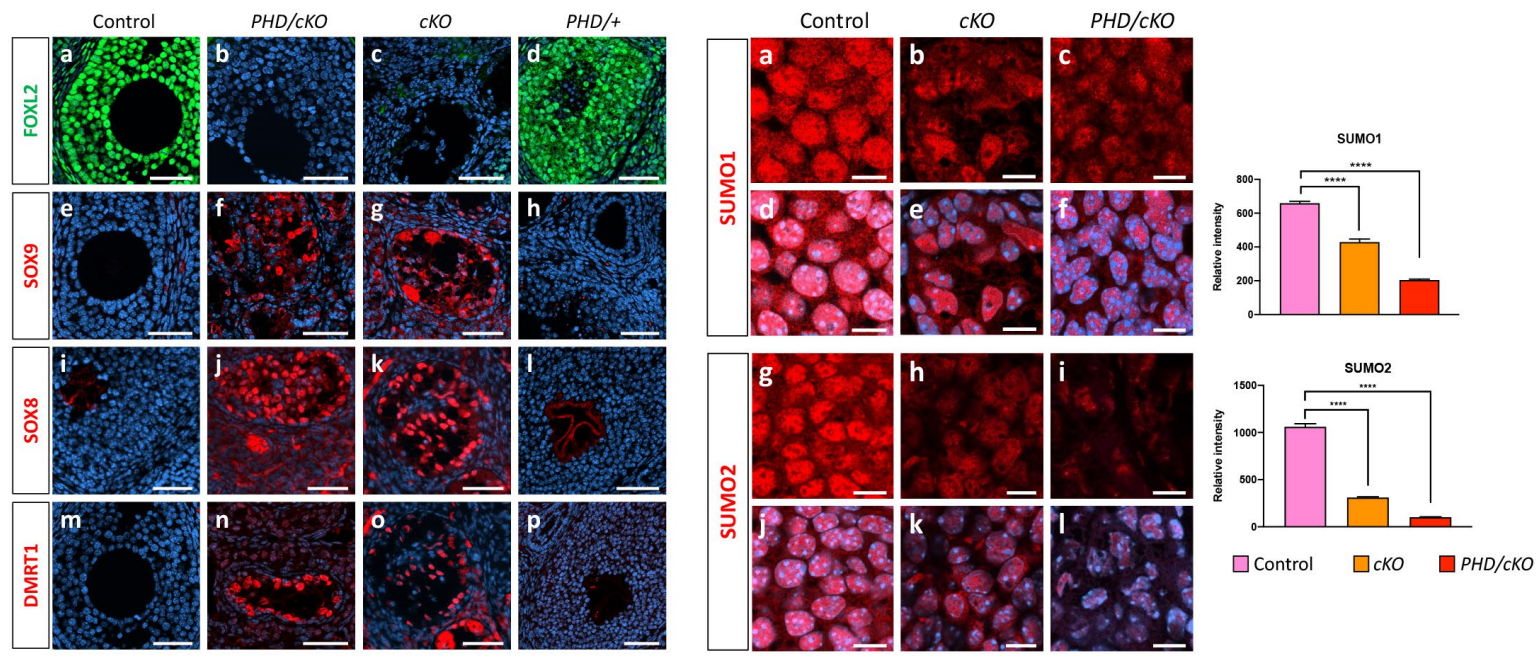

Fig. 4: Loss of TRIM28 SUMO-E3 ligase activity in granulosa cells phenocopies Trim28

conditional knock-out. (A). Schematic of the SUMO pathway with TRIM28 E3-SUMO ligase

activity. After their proteolytic maturation by sentrin-specific proteases (SENPs), SUMO2 and 3

diverge by only one residue, making them indistinguishable by antibodies, thus they are currently 
referred to as SUMO2. SAE1/2, SUMO-activating enzyme 1/2. (B). RT-qPCR analysis of femaleand male-specific genes in 8-week-old Trim $28^{c K O}$, Trim $28^{P H D / c K O}$, Trim $28^{P H D /+}$ and control ovaries. Data are the mean $\pm \operatorname{SEM}(\mathrm{n}=6) .{ }^{*} * * * P<0.0001, * * * P<0.001, * * P<0.01, * P<0.1$ (ANOVA with Tukey's multiple comparisons test). (C). FOXL2 is expressed in control (a) and $\operatorname{Trim} 28^{P H D /+}$ (d) ovaries, but not in Trim $28^{P H D / c K O}$ and Trim $28^{c K O}$ ovaries (b and c). Like in Trim2 $8^{c K O}$ ovaries (g, k, and o), SOX9, SOX8 and DMRT1 are expressed in pseudo-tubules of $\operatorname{Trim} 28^{P h d / c K O}$ ovaries (f, j, and n), but not in control (e, i, and m) and Trim $28^{P H D /+}$ (h, 1, and p)

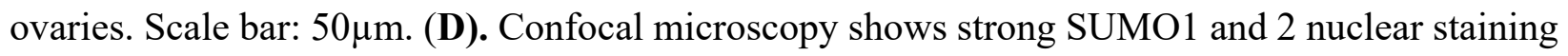
in granulosa cells of control ovaries. The staining intensity is markedly decreased in Trim $28^{c K O}$ (cKO: b, e, h, and k) and Trim2 $8^{P H D / c K O}(P H D: \mathrm{c}, \mathrm{f}, \mathrm{i}$, and 1) ovaries. Right panels: quantification of SUMO1 and SUMO2 signal intensity relative to DNA staining. Data are the mean \pm SEM $(\mathrm{n}=100) ; * * * * P<0.0001$ (ANOVA with Tukey's multiple comparisons test). 
Fig 5: Genome-wide SUMOylation changes in Trim $28^{c K O}$ and Trim $28^{\text {Phd/cko }}$ ovaries. (A) and

(B). Upper table shows the number of SUMO1 and SUMO2 peaks significantly decreased (Log2

Fold Change <1; hypo-SUMOylated; blue), or increased (Log2 Fold Change >1; hyperSUMOylated; red) in Trim $28^{c K O}$ and $\operatorname{Trim} 28^{P h d / c K O}$ ovaries compared with controls. Lower panels: heatmaps of SUMO1, SUMO2 and TRIM28 ChIP-seq reads from control (Cont), Trim28 $8^{c K O}$ (cKO) and Trim $28^{P h d / c K O}(P H D)$ ovaries mapped ( $\pm 1 \mathrm{~kb}$ from the center) to SUMO1 and SUMO2 hypo-SUMOylated peaks (blue traces) and to SUMO1 and 2 hyper-SUMOylated peaks (red traces). (C). Pie charts showing that genes that are downregulated and display SUMOylation changes in Trim $28^{c K O}$ ovaries are preferentially hypo-SUMOylated, while upregulated genes with SUMOylation changes are preferentially hyper-SUMOylated. Number of genes are between brackets. Genes are listed in Data S7. (D). Examples of SUMOylation status (SUMO1 and 2) in control, Trim $28^{c K O}(\mathrm{cKO})$ and Trim $28^{P h d / c K O}(P H D)$ ovaries of genes the expression of which is altered in Trim $28^{P h d / c K O}$ ovaries. Light blue and red, regions significantly hypo-SUMOylated and hyper-SUMOylated, respectively, in mutants. 